\title{
L'économie de l'enseignement supérieur au Québec au cours de la dernière décennie*
}

\author{
CLÉMENT LEMELIN†
}

\section{RÉSUMÉ}

En plus d'établir une bibliographie des principaux travaux en économie de l'enseignement supérieur au Québec publiés en français au cours des dix dernières années, l'article qui suit contient une brève présentation de cette littérature. Ces travaux sont regroupés sous cinq sous-titres: les techniques de planification, l'effort de la société québécoise, l'impact de la scolarité sur le marché du travail, la demande privée d'enseignement et la syndicalisation des professeurs. L'article se termine par quelques suggestions de recherches.

\begin{abstract}
This article surveys the economics of higher education in francophone Quebec during the 1970's. The main contributions are presented under the five following headings: attempts at planning, comparative studies of expenditures on higher education, the impact of education on the labor market, the private demand for education and collective bargaining. Areas of future research are also suggested.
\end{abstract}

Cet article, qui constitue le résumé d'une communication présentée au congrès de 1981 de la SCEES, esquisse un bilan des principaux travaux effectués en économie de l'enseignement supérieur au Québec et publiés en français au cours de la période 1970-1981. Ainsi que l'ont suggéré les organisateurs de ce congrès, le champ de l'économie de l'enseignement supérieur inclut les travaux qui portent sur la planification de l'enseignement et le syndicalisme des enseignants, mais exclut les travaux dont l'objet d'étude est l'impact des facteurs ou des techniques

\footnotetext{
* Nous tenons à remercier Guy Girard et Normand Proulx, dont tous les commentaires et suggestions n'ont cependant pas été retenus.

†Département de Science Economique, Université đu Québec à Montréal
} 
d'enseignement. Pour des raisons d'espace, la bibliographie que l'on retrouve à la fin de ce texte ne comprend que les titres auxquels il est fait référence dans le corps de l'article. ${ }^{1}$

\section{LE QUÉBEC DES ANNÉES 1970}

Les travaux qui sont recensés ici ont été souvent effectués à la demande d'organismes oeuvrant dans le secteur de l'enseignement supérieur et, pour mieux en saisir le sens, il est utile de comprendre certains problèmes de la société québécoise. En 1970, le Québec digère encore diverses réformes de son système d'enseignement; c'est une période de rodage au cours de laquetle les Québécois partagent encore la vision optimiste que l'on trouve dans le Rapport de la Commission d'Enquête sur l'enseignement dans la Province de Québec et selon laquelle l'enseignement constitue un investissement rentable pour l'étudiant et pour la société. Tout semble encore possible et s'il y a quelque chose à planifier, c'est la croissance. Au fur et à mesure que la décennie s'écoule, les évaluations, les consolidations et les remises en question succèdent aux réformes et aux innovations. Les priorités changent et l'éducation ne semble plus porteuse des espoirs de développement. La société québécoise trouve de plus en plus difficile de supporter le rythme de croissance des dépenses publiques. Rappelons ici qu'au Québec, les droits de scolarité ont été abolis dans les collèges, qu'ils sont gelés depuis 1968 dans les universités et que les taux de fréquentation scolaire continuent de croître, contrairement au reste de l'Amérique du Nord. La planification doit maintenant s'effectuer dans un contexte de restriction budgétaire. C'est ainsi que les travaux visant à élaborer des techniques raffinées de planification de l'enseignement ont cédé petit à petit la place à des exercices, peut-être moins sophistiqués mais tout aussi utiles, d'évaluation de l'effort de la société québécoise dans le domaine de l'enseignement supérieur. Les deux premières parties de la section suivante porteront donc sur les techniques de planification et sur les évaluations des efforts de la société québécoise.

Il existe cependant plusieurs façons d'évaluer le caractère optimal des dépenses d'enseignement. L'une des plus populaires consiste à déterminer le taux de rendement de ces dépenses. Nous avons rassemblé, dans la troisième partie de la prochaine section, les divers travaux qui ont tenté de cerner l'impact des études sur le marché du travail avant de présenter quelques travaux qui tentent de préciser la nature des principaux déterminants de la demande d'éducation.

Un des développements les plus intéressants de la vie universitaire québécoise au cours des années 1970 demeure la syndicalisation du corps enseignant. Aussi notre recension des travaux se terminera-t-elle par une brève partie qui présentera des travaux qui ont tenté d'évaluer l'impact du syndicalisme enseignant.

\section{LES TRAVAUX EN ÉCONOMIE DE L'ENSEIGNEMENT SUPÉRIEUR}

Le problème de base en économie de l'enseignement supérieur au début des années 1970 en était un de définition des techniques de planification de l'enseigne- 
ment. La mise en place d'une structure d'enseignement supérieur comprenant collèges et universités, la création d'une université à constituantes multiples, l'Université du Québec, dont les retombées régionales on été analysées récemment par Polèse et Léger (1970), l'augmentation rapide de la population estudiantine et des coûts de l'enseignement, ainsi que l'implication toujours plus grande de l'Etat dans ce secteur, ont rendu nécessaires une évaluation des activités des collèges et des universités, une meilleure coordination de ces institutions et une discussion plus systématique des objectifs à poursuivre et des moyens pour les atteindre.

\section{Les techniques de planification}

Parler de planification de l'enseignement supérieur au Québec au début des années 1970, c'est parler de l'Opération Système d'Information de Gestion Universitaire (SIGU), lancée par le Ministère de l'Education du Québec en 1969 et coordonnée par le Comité d'Elaboration d'un S.I.G.U. (CESIGU). L'objectif principal était "d'assurer la concordance et la compatibilité de l'information à la disposition des diverses composantes du système universitaire". Le système d'information uniformisée devait recouvrir les secteurs suivants: étudiant, personnel enseignant et non enseignant, finance, programme de cours et de recherche, ressources physiques.

Même si les résultats de cette Opération ne furent pas à la mesure des ambitions qui lui donnèrent naissance et même s'il est impossible de tracer ici la liste de tous les travaux qui ont été effectués dans le cadre de cette Opération, l'effort mérite d'être souligné. Il fut notamment l'occasion d'élaborer des modèles de prévisions d'effectifs étudiants (Florian \& Guérin, 1971). Guérin (1973, 1974, 1975) devait d'ailleurs revenir à plusieurs reprises au cours de la période sur ce sujet qu'abordent également Bordeleau et Légaré (1970) ainsi que Légaré (1971, 1972) et Girard (1976).

La planification de l'enseignement est souvent confondue avec une technique particulière, les prévisions de besoins de main-d'oeuvre. La longue monographie de Contandriopoulos (1971) constitue sans conteste l'exemple le plus original d'élaboration par un chercheur individuel d'une stratégie de planification en fonction des besoins de main-d'oeuvre. L'effort de Contandriopoulos est cependant demeuré théorique, n'ayant pas débouché sur une application empirique. Le modèle donne prise moins facilement aux critiques habituelles des tenants d'une approche plus néo-classique, puisque l'univers analysé est divisé en divers secteurs soumis à des règles de fonctionnement différentes; il devient ainsi plus flexible. Ces divers secteurs sont analysés à l'aide de méthodes diverses et le modèle de Contandriopoulos devient rapidement éclectique, ce qui n'est pas nécessairement un défaut. Enfin, le modèle tient compte de certaines informations de marché puisqu'il incorpore des clignotants qui indiquent la nécessité d'une intervention nouvelle de la part du planificateur.

Dans le milieu de la décennie, le Ministère de l'Education du Québec a préféré adopter une stratégie des petits pas et il a lancé trois opérations sectorielles, en sciences appliquées, en sciences de la santé et en sciences fondamentales. Après 
avoir fait le bilan des programmes, des ressources, des clientèles et des débouchés se rapportant à chacune des disciplines qui étaient couvertes par les opérations, on cherche à esquisser un plan de développement pour les années à venir (Québec, 1973, 1975, 1976). Si l'on se fie à l'évaluation que fait Lapointe (1976) de l'Opération sciences fondamentales, les objectifs de ces opérations ne furent atteints que bien partiellement. Ces opérations ont souffert de leur caractère sectoriel, ne pouvant prendre appui sur des objectifs plus globaux. De plus, à l'intérieur d'une même opération, les disciplines ont souvent été évaluées par des chercheurs qui travaillaient isolément; les rapports particuliers trahissent souvent une hétérogénéité de méthode d'analyse, voire de philosophie; enfin, il semble bien que plusieurs des recommandations doivent rester lettre morte, le suivi devant être assuré par les universités individuelles.

Cette expérience des opérations sectorielles a été reprise récemment par des études mini-sectorielles dont l'un des objectifs est la réorientation des programmes; science politique, toxicologie, géologie et génie géologique, informatique, nutrition et travail social ont été tour à tour analysés.

Un autre exemple de tentative de planification, enfin, nous est donné par la vaste étude du Conseil des Universités portant sur les objectifs généraux de l'enseignement supérieur et les grandes orientations des établissements universitaires (Québec, 1972,1973 et 1976). Cette opération a la caractéristique d'avoir intégré les diverses institutions d'enseignement tout au long de la définition des objectifs et des orientations. Mais, encore une fois, il ne semble pas que les résultats de la démarche aient été probants eu égard à l'objectif de planification.

\section{Les évaluations de l'effort de la société québécoise}

L'ampleur de l'effort qu'a consenti la société québécoise dans le domaine de l'enseignement supérieur au cours de la période devait bien vite inciter plusieurs chercheurs à comparer cet effort avec celui des sociétés voisines. Farine (1970), la CREPUQ (1978) et la Direction Générale de l'Enseignement Supérieur (Québec, 1980) s'interrogent tour à tour sur les différences dans les dépenses effectuées au Québec et en Ontario. Il est certain que le recours, pour le Québec, à un tel critère d'efficacité ne peut emporter l'adhésion de tous. Désy (1977) montre combien il est difficile de comparer des systèmes d'enseignement supérieur aussi différents que ceux du Québec et de l'Ontario. Toute comparaison visant à mesurer l'efficacité et les coûts unitaires repose aussi très lourdement sur la pondération à accorder aux étudiants à temps partiel et à temps complet puisque les premiers sont relativement plus nombreux au Québec. De plus, la seule comparaison Québec-Ontario risque de faire oublier une autre différence entre Québécois francophones et anglophones. Enfin, il existe certainement quelques Ontariens qui seront portés à sourire au vu d'une volonté d'imiter à tout prix l'expérience ontarienne.

Bélanger (1970, 1972, 1977), Proulx (1973) et Lemelin (1979) tentent d'expliquer pourquoi l'éducation coûte si cher. Parmi les raisons moins évidentes retenues, mentionnons les effets d'imitation et d'incitation que créent l'apparte- 
nance à la Confédération canadienne et le système de financement de l'enseignement post-secondaire, le peu d'incitation à réduire les coûts de la part d'organisations à but non lucratif et le développement soudain et rapide des secteurs tertiaire et public, grands utilisateurs de main-d'oeuvre qualifiée et francophone au Québec.

Selon la CREPUQ (1978), ce qui distingue le Québec de l'Ontario, c'est beaucoup plus l'effort de l'Etat que celui de la société prise dans son ensemble. Faire cette distinction, c'est reposer le problème du financement de l'enseignement supérieur. Bélanger (1973) et Lemelin (1980) analysent la répartition du fardeau du financement des études universitaires entre les étudiants et les contribuables. Parce que les droits de scolarité à l'université ont été gelés et, aussi, parce que l'inflation a diminué la charge que représentent pour les étudiants des emprunts dont les intérêts sont payés par l'Etat pendant la période d'étude, ce fardeau s'est allégé pendant toute la période dans le cas des étudiants.

Sur le sujet du financement de l'enseignement supérieur, Farine (1971) opte pour un fonds d'autofinancement des frais de scolarité par lequel les étudiants pourraient être appelés à financer une plus grande partie des coûts directs. Drakos (1973) favorise lui aussi un système qui ferait place à des emprunts plus considérables et remboursables en fonction du revenu futur. Cette possibilité est aussi évoquée par Farine, Proulx \& Lemelin (1979). Plus près de nous, Lemelin (1980) favorise des mesures sélectives plutôt qu'universelles: dans un univers de rareté de plus en plus grande, une plus grande accessibilité passerait par une augmentation des droits de scolarité accompagnée d'un système plus généreux de prêts et bourses, ces dernières étant attribuées après prise en considération des ressources financières de l'étudiant. Dans ce dernier cas, ce sont des considérations de justice sociale qui sont invoquées en faveur de mesures sélectives. En effet, le financement public de l'enseignement supérieur semble se solder par un transfert de ressources favorisant les mieux nantis.

Un tel argument risque de ne pas emporter l'adhésion de plusieurs pour qui les problèmes fondamentaux du Québec sont sa situation de retard dans son effort de scolarisation et sa position particulière qui l'oblige souvent à ne compter que sur ses propres moyens. On peut relever plusieurs études qui décrivent les principales caractéristiques de la main-d'oeuvre hautement qualifiée du Québec. S'inspirant d'études que l'IIEQ (1971) et Girard (1973) avaient effectuées précédemment pour la Commission d'enquête sur la situation de la langue française et sur les droits linguistiques, Girard, Otis et Proulx (1978) montrent combien les Québécois francophones sont sous-représentés dans certains secteurs et combien le Québec dépend aujourd'hui encore de l'étranger. Farine (1974), quant à lui, insiste sur le fait que la situation diffère d'un secteur à l'autre, les francophones ayant tendance à se retrouver dans des entreprises publiques ou para-publiques. Lacroix et Proulx (1973) s'intéressent eux aussi au problème de mobilité: à la fin de leur recherche, ce qui aurait pu passer pour un don s'avère une opération rentable; en effet, si la formation d'étudiants venant d'autres provinces entraîne des coûts, pour la province d'accueil, la perte n'est pas nette 
puisqu'à la fin de leurs études plusieurs étudiants demeurent dans cette dernière province, qui n'aura pas eu à défrayer les coûts de la formation pré-universitaire. Au Québec, ces problèmes de mobilité importent beaucoup plus dans le cas des Québécois anglophones. Enfin, soulignons une autre étude de Girard, Otis et Proulx (1977) qui posent le problème de la dépendance du Québec face à l'extérieur dans le domaine de la recherche scientifique.

\section{L'impact de l'enseignement sur le marché du travail}

Le coût très élevé de l'enseignement supérieur a bien vite amené les économistes à s'interroger sur la pertinence de la formation eu égard à sa relation avec le marché du travail. Même si les calculs de taux de rendement constituent la façon la plus précise d'évaluer l'efficacité de l'enseignement supérieur, il est difficile de relever plusieurs exemples de calcul effectué spécifiquement à cette fin. Tout au plus peut-on relever la recherche d'Otis (1976) qui infère des taux de rendement attendus à partir des attentes des étudiants du Département de sciences économiques de l'Université de Montréal. En 1974, les taux de rendement moyen et médian étaient égaux à environ $9 \%$ et ils diminuaient avec le niveau de scolarité.

En fait, c'est beaucoup plus dans le cadre d'études visant à déterminer les causes des disparités de revenus qu'on est parvenu à obtenir des estimés de taux de rendement. Utilisant une fonction de détermination des revenus élaborée par Mincer, ${ }^{2}$ Tremblay (1976), Lacroix et Lemelin (1980) ainsi que Lacroix, Lemelin et Robillard (1978) évaluent les disparités de revenu au sein de la maind'oeuvre hautement qualifiée du Canada. Les taux de rendement, qui diminuent avec le niveau de scolarité, ne semblent guère différer des taux qu'on a trouvés aux Etats-Unis. Le rôle du niveau de scolarité est beaucoup moins important que le rôle de l'expérience lorsqu'il s'agit d'évaluer les disparités de revenu. De plus, le champ de spécialisation joue un rôle important que la durée des études et l'expérience ne peuvent expliquer. Enfin, ces deux dernières variables jouent un rôle différent d'un domaine de spécialisation à l'autre.

Lacroix et Vaillancourt (1980) reprennent cette étude mais avec un échantillon limité aux seuls Québécois. Les principales conclusions s'y trouvent confirmées. Lacroix et Vaillancourt vont cependant plus loin et s'interrogent aussi sur la rentabilité de la maîtrise du français ou de l'anglais, prolongeant ainsi la réflexion amorcée par Migué (1970) et Raynauld et Marion (1972).

L'étude de l'impact de la scolarité sur les revenus n'est d'ailleurs plus l'apanage des économistes puisque des sociologues comme Allarie, Bernard et Renaud (1979) s'y intéressent.

L'étude de la corrélation scolarité-revenu n'est qu'une façon parmi plusieurs d'étudier la pertinence de la formation post-secondaire, l'étude de la corrélation scolarité-chômage en constituant une autre. Au cours de ces dernières années, plusieurs économistes se sont interrogés sur la réalité que pourrait recouvrir l'idée de chômage des jeunes diplômés. Lemelin (1979) et Saint-Laurent (1979) affirment tour à tour que le problème en est un de sous-utilisation des compétences, présumées ou réelles, beaucoup plus que de chômage proprement dit. 
Girard, Gauthier et Vinet (1978) indiquent cépendant qu'il ne faut pas sousestimer les anticipations et les espoirs déçus des jeunes. Il semble donc que s'il existe un problème d'emploi chez les jeunes diplômés, ce problème tient au fait que ces derniers sont jeunes beaucoup plus qu'à celui qu'ils sont diplômés. Le chômage des jeunes diplômés serait un sous-problème d'un problème bien plus grave, le chômage des jeunes. Dans ce qui semble devoir être pour quelque temps le point final au débat, Cousineau (1980) montre bien le caractère relatif et souvent transitoire du chômage des nouveaux diplômés.

Une troisième façon d'évaluer la pertinence de la formation post-secondaire consiste à étudier l'adéquation formation-emploi. A partir, notamment, de la banque de données créée lors de l'Enquête sur la main-d'oeuvre hautement qualifiée et d'une autre créée à la suite d'un sondage effectué auprès des diplômés de l'Université de Montréal, Farine (1974, 1975a, 1975b, 1979, 1980), Farine \& Knowles (1976), ainsi que Farine \& Proulx (1975) se sont longuement interrogés sur les liens qui se tissent entre la formation et l'emploi. Comme on peut s'y attendre, il ressort que, sauf dans le cas de quelques disciplines menant à l'exercice de professions dominées par des corporations professionnelles, les liens s'avèrent souvent ténus en ce sens précis que la plupart des disciplines ne mènent pas à l'exercice d'une seule profession. Girard, Otis \& Proulx (1978) arrivent aux mêmes conclusions.

C'est cette même pertinence de la formation que tentent d'évaluer les multiples opérations de relance effectuées par les organismes gouvernementaux et les maisons d'enseignement, et au cours desquelles on s'interroge sur ce qu'il est advenu des étudiants qui ont mis un terme à leurs études. L'énumération des relances effectuées pour le compte d'établissements particuliers pouvant s'avérer fastidieuse, contentons-nous, à titre d'illustration, de renvoyer le lecteur aux travaux de Guérin (1979) et Vergès-Escuin (1977).

Quant au Ministère de l'Education du Québec, il mène des opérations relances depuis 1973. A chaque année, il collige des renseignements sur l'insertion des sortants des secteurs professionnels des écoles secondaires et des collèges sur le marché du travail. La monographie de Darveau (1978) décrit très bien la richesse des données ainsi recueillies. Malheureusement, les travaux auxquels ont donné naissance ces opérations ont dû se contenter d'être descriptifs plutôt qu'analytiques; ce qu'il faut déplorer ici, c'est que cette mine de renseignements n'ait pas été davantage exploitée. Plus récemment, le Ministère de l'Education effectuait aussi une relance auprès des diplômés d'université. Audet (1979) nous en décrit les principaux résultats; ici aussi il semble bien qu'on soit en droit d'attendre encore plus d'analyse des données recueillies.

\section{La demande privée d'enseignement}

En économie de l'éducation, les tenants de l'analyse du taux de rendement ne se contentent pas de calculer des taux de rendement; ils prétendent également que les individus sont sensibles à des considérations de rentabilité. Les travaux visant à identifier les déterminants de la demande d'éducation sont cependant plus rares. 
Lemelin et Otis (1978), ainsi que Berrouard (1979), cherchent à isoler les facteurs qui déterminent le choix d'une discipline. Contrairement à ce que laissent entendre les plus étroites des interprétations économiques dominantes, les disparités de revenu de travail associées aux diverses disciplines ne semblent pas constituer un déterminant important du choix de carrière. Les possibilités d'emploi de même que le caractère intéressant des emplois auxquels mène la discipline paraissent jouer un rôle prédominant; si tel est le cas, l'université conserve, aux yeux des étudiants, son rôle de préparation au marché du travail.

Plusieurs économistes sont prêts à reconnaître, cependant, que d'autres facteurs que ceux qui sont associés aux coûts privés et au marché du travail peuvent influer sur la décision de poursuivre ses études ou de choisir un champ de spécialisation. Dans leurs efforts visant à identifier les variables qui influencent la décision des collégiens de poursuivre leurs études à l'université, Houle et Ouellet (1981) insistent, quant à elles, sur l'importance de l'environnement familial.

La plupart de ces études supposent une démarche linéaire de la part des étudiants qui s'intègrent au système d'enseignement à cinq ans et qui le quittent petit à petit, au fur et à mesure que le coût marginal de la poursuite des études finit par rejoindre le bénéfice marginal. Raisonner ainsi, c'est trop souvent passer sous silence d'autres stratégies par lesquelles on quitte et on réintègre le système d'enseignement. C'est faire abstraction de l'éducation des adultes ou de l'éducation récurrente, sujets d'étude qui pourraient s'avérer importants au Québec puisque la place relative réservée aux étudiants adultes ou à temps partiel y est beaucoup plus grande qu'au Canada.

Lizée et Lizée (1978), de même que Daoust, Amyot, Fortin et Harvey (1978) font de l'éducation des adultes le premier pas vers l'instauration d'un véritable système d'éducation permanente et d'une politique de démocratisation de l'enseignement. On peut cependant se demander si l'éducation des adultes au niveau post-secondaire a toutes les caractéristiques d'une démarche de démocratisation: ces adultes ne constituent-ils pas un groupe déjà favorisé au sein de l'ensemble de la population? Lemelin (1980) indique néanmoins que l'origine sociale des étudiants à temps partiel en fait des gens nettement moins favorisés que leurs camarades à temps complet.

Jusqu'ici, bien peu d'études sur l'éducation des adultes ont été effectuées à partir de considérations d'efficacité. Raynauld (1975) semble être le seul à avoir soulevé les questions qui sont traditionnellement du domaine de l'économie de l'éducation: qu'en est-il de l'influence de l'expérience de travail sur l'apprentissage dans les maisons d'enseignement? Qu'en est-il de l'obsolescence des connaissances acquises là? De toute évidence, les recommandations visant à transformer le système d'éducation afin qu'il s'inspire du principe de l'éducation permanente devront fournir des réponses à ces questions.

\section{La syndicalisation des enseignants}

L'expansion du système universitaire québécois au cours des années 1970 s'est réalisée en même temps que se généralisait la syndicalisation des professeurs. 
La naissance de ce type de syndicalisme semble avoir fait couler beaucoup plus d'encre que la pratique quotidienne de ces activités. C'est ainsi que le gros des réflexions des économistes semble avoir été publié dans la première partie de la décennie (Chung, 1973; Roy, 1973; Bélanger, 1975; Boivin, 1975; Desgagné et Miller, 1975).

Comme l'indique le recueil de textes publié par un collectif d'universitaires (1977), c'est beaucoup plus la répartition des pouvoirs à l'intérieur de l'université que les conditions matérielles de travail des professeurs qui semble avoir été objet de recherche.

Tout récemment cependant, Marois (1981) a entrepris d'évaluer de façon systématique l'impact de la syndicalisation des professeurs sur la structure de rémunération. De son analyse fouillée, il ressort que la présence d'un syndicat s'accompagne d'une diminution de la dispersion des salaires autour des échelles; de plus, s'il n'est pas sûr que le syndicalisme réduise les écarts entre les revenus des hauts et des bas salariés, il semble bien que le syndicalisme réduise les écarts interdisciplinaires de revenu. Au total, la syndicalisation semble bien avoir eu un impact favorable, mais pas trop considérable, sur le niveau moyen de rémunération et cet impact aurait été approprié en grande partie par ceux qui se trouvent dans des disciplines moins favorisées. Cette étude semble bien devoir devenir un ouvrage de référence obligatoire pour quiconque veut connaître les déterminants des salaires des professeurs d'université, d'autant plus que les travaux publiés sur le sujet sont très rares.

\section{BILAN ET PERSPECTIVES}

Rien ne sert de cacher notre perplexité au terme de notre survol de la littérature. Si l'on peut parler de vitalité, l'on doit aussi noter la grande dispersion des efforts. Faute d'une structure adéquate d'information, bien des travaux sont ignorés de la communauté scientifique. Cette lacune n'est comblée que bien imparfaitement par l'Inventaire de recherches de l'A.U.C.C., la Bibliographie de l'enseignement supérieur du Professeur Harris et le nouveau Répertoire des projets de recherche du Ministère de l'Education du Québec. Nous sommes persuadés que l'impossibilité de rassembler rapidement toutes les recherches portant sur l'enseignement supérieur a grandement réduit l'efficacité de la Commission d'étude sur les universités.

Plutôt que d'insister sur la nécessité d'une structure qui permettrait de mettre en contact les divers chercheurs qui oeuvrent dans le domaine et qui favoriserait ainsi une réflexion plus poussée, nous préférons plutôt suggérer des objets de recherche qui nous semblent prioritaires.

1. D'abord, le temps nous semble venu de faire un bilan des diverses relances effectuées tant par le Ministère de l'Education du Québec que par des chercheurs oeuvrant dans diverses maisons d'enseignement. Il y a beaucoup plus à retirer de ces relances que des indicateurs de conjoncture sur le marché du travail. Qu'en est-il notamment de l'efficacité du système d'enseignement eu égard à l'objectif d'intégration sur le marché du travail? Une réponse claire à cette question pourrait 
éclairer les débats à venir sur la façon de financer l'enseignement post-secondaire. Ce bilan devrait aussi tenir compte des deuxième et troisième relances auprès des sortants d'université et de la relance auprès des relancés qu'a effectuées le Ministère de l'Education du Québec, dont on sait encore peu de choses et qui, dans le dernier cas, permettra de mieux évaluer le caractère durable de l'influence d'une scolarité plus poussée.

2. Si l'on songe à accorder une importance plus grande au taux de rendement comme un des critères de gestion de l'enseignement post-secondaire, ou à adopter un système de droits de scolarité qui reflètent davantage les coûts de l'enseignement, ou à utiliser une méthode de financement public des universités qui ne soit pas la méthode historique, il nous semble important d'entreprendre des études systématiques des coûts des diverses activités des maisons d'enseignement. Ces dernières sont peut-être bien' équipées pour identifier ces coûts rapidement (Vallerand, 1977), mais, à notre grand étonnement, nous n'avons trouvé aucune trace de résultats empiriques sur ce sujet. Cela pourrait tenir au caractère trop souvent confidentiel des études effectuées par les services de planification ou de recherche institutionnelle des universités, dont la diffusion des résultats est fort restreinte. Quant à nous, il nous semble que pour déboucher sur des résultats fiables, il faudra procéder également à une sérieuse étude de l'allocation du temps des professeurs.

3. Compte tenu de l'importance qu'acquiert déjà la population des étudiants à temps partiel ou adultes dans le système québécois d'enseignement supérieur, il nous semble que les chercheurs devront s'intéresser davantage à ces étudiants, d'autant plus que leur importance relative est appelée à croître dans les années à venir. Le Conseil des Universités pilote déjà un projet de recherche sur le sujet. Outre les questions habituelles portant sur le problème de l'accessibilité, deux autres problèmes nous semblent particulièrement intéressants: le premier porte sur une comparaison des coûts de formation des étudiants à temps partiel et à temps complet; le deuxième relève plus particulièrement de ce qu'on appelle la micro-économie de l'enseignement et porte sur les déterminants de l'apprentissage en même temps que sur les effets plus ou moins durables de cet apprentissage. L'êtude de l'impact de l'expérience de travail ainsi que du rythme de l'obsolescence des connaissances nous semble primordiale. Une réponse à ces questions nous mènerait peut-être aux mêmes conclusions que les tenants les plus convaincus d'un système d'éducation permanente mais à partir de considérations différentes et tout aussi valables.

4. Si quelque chose nous irrite dans toute la littérature en économie de l'éducation, c'est bien le divorce qui sépare les études d'accessibilité et de rentabilité. Il semble bien y avoir ici un terrain possible de rencontre pour économistes et sociologues. De façon plus spécifique, nous connaissons plus d'un économiste qui considèrent avec envie la richesse des données recueillies dans le cadre du projet ASOPE (Québec, 1979). L'existence de données longitudinales constituant une denrée rare, cette banque de données devrait être exploitée au maximum. A première vue, il nous semble que ces données pourraient permettre de mieux 
comprendre et de décortiquer les influences de l'école et du milieu familial sur le marché du travail. Ce sont là des questions primordiales en économie de l'éducation. De la même façon, on peut se demander s'il ne serait pas possible de mieux comprendre l'influence de l'origine sociale sur la rentabilité des études. Enfin, toutes ces informations pourraient nous permettre de mieux comprendre un des grands problèmes des sociétés contemporaines, les difficultés d'intégration des jeunes sur le marché du travail.

\section{NOTES}

1. Le lecteur intéressé pourra toujours consulter le tex te plus complet de notre communication (Lemelin 1981).

2. Voir Mincer, J. Schooling, experience and earnings. New York: National Bureau of Economic Research, 1974.

\section{REFERENCES}

Allarie, A., Bernard, P. \& Renaud, J. Qui s'instruit s'enrichit? Possibles, 1979, 3 (3/4), 13-33.

Audet, M. Relance à l'université. Québec: Ministère de l'Education, Direction des politiques et plans, Gouvernement du Québec, 1979.

Bélanger, G. L'université, organisme à but non lucratif. Revue d'économie politique, 1977, $87(4), 574-590$.

Bélanger, G. La syndicalisation des professeurs d'université. Revue de l'ACELF, 1975.

Bélanger, G. Le financement de l'enseignement supérieur au Québec. Actualité Economique, $1973,49(4), 467-499$.

Bélanger, G. Le secteur public, un budget croissant pour des services constants. Revue Economique, 1972, 23 (1), 70-85.

Bélanger, G. Universités et hôpitaux: Où allons-nous? Canadian Banker, 1970, 77 (2), 30-32.

Berrouard, J.M. L'explication des choix de la discipline d'études a l'université: analyse empirique pour le Québec. Montréal: rapport de recherche, Département de sciences économiques, Université de Montréal, 1979.

Boivin, J. La négociation collective chez les professeurs d'université. Quelques cas. Relations Industrielles, 1975, 30 (4), 674-706.

Bordeleau, J. \& Legaré, J. Les méthodes de prévision de la population étudiante au niveau universitaire dans le contexte québécois, Montréal: Conférence des recteurs et principaux des universités du Québec, 1970.

Chung, J. Syndicalisme des professeurs d'université. Quelques réflexions. Relations Industrielles, 1973, 28 (2), 325-342.

CREPUQ (Conférence des recteurs et des principaux des universités du Québec). Analyse de quelques indicateurs du niveau de développement du système d'enseignement supérieur au Québec, de l'effort relatif de la société et du gouvernement et de la productivité des universités québécoises. Montréal: CREPUQ, 1978.

Contandriopoulos, A.P. Prévision des besoins en main-d'oeuvre hautement qualifiée et planification de l'éducation. Montréal: Conférence des recteurs et des principaux des universités du Québec, 1971. 
Cousineau, J.M. Le marché du travail des diplômés universitaires au Québec. Coll. dossiers, Québec: Conseil des Universités, Gouvernement du Québec, 1980.

Daoust, G. \& P. Bélanger L'université dans une société éducative. Montréal: Presses de l'Université de Montréal, 1974.

Daoust, G., Auyot, P., Fortin, A., Harvey, P. Education et travail. Montréal: Hurtubise HMH, 1978.

Darveau, J.G. Relance. Etude de la situation des sortants de l'enseignement professionnel, aux niveaux secondaire et collégial pour la période de 1973 à 1976. Québec: Gouvernement du Québec, Ministère de l'Education, Direction des politiques et plans, 1978.

Desgagné, A. \& Miller, R. L'université et la syndicalisation de ses professeurs. Québec: L'Editeur officiel, 1975.

Désy, J. Remarques méthodologiques sur la participation du Québec à l'enseignement postsecondaire. Revue canadienne de l'enseignement supérieur, 1977, 7 (3), 59-71.

Drakos, G. L'économique, la politique de l'enseignement universitaire et les frais de scolarité. Actualité Economique, 1973, 49 (3), 435-440.

Farine, A. Aspects de l'expérience universitaire et de la carrière professionnelle des étudiants: les diplômés et les étudiants qui ont abandonné leurs études pour 1973.74 à l'U de $M$, en sciences pures et en sciences humaines. Montréal: Faculté des sciences de l'éducation, Université de Montréal, 1975.

Farine, A. Etude comparée des subventions provinciales à l'enseignement supérieur. Actualité Economique, 1970, 46 (3), 521-531.

Farine, A. Fonds d'auto-financement des frais de scolarité. Bulletin de l'A.C.P.U., 1971, 19 (3), 13-18.

Farine, A. La perception des employeurs des liens qui existent entre la formation et l'emploi. Montréal: Faculté des sciences de l'éducation, Université de Montréal, 1980.

Farine, A. Les diplômés de l'Université de Montréal sur le marché du travail. Montréal: Université de Montréal, CRDE, 1974.

Farine, A. Les diplômés en sciences de l'éducation: étude sur la concordance entre l'emploi et la formation universitaire. Montréal: Faculté des sciences de l'éducation, Université de Montréal, 1975.

Farine, A. Les liens entre la formation et l'emploi en France, en Allemagne, et en Italie. Montréal: Faculté des sciences de l'éducation, Université de Montréal, 1979.

Farine, A. \& Knowles, R. La formation et l'emploi des diplômés en beaux-arts, en humanités et en sciences sociales du Canada. Revue canadienne de l'enseignement supérieur, 1976,6 (2), 11-21.

Farine, A. \& Proulx, P.P. La correspondance entre la formation et l'emploi chez un échantillon des sortants de l'université. Actualité Economique, 1975, 51 (3), 472-483.

Farine, A., Proulx, P.P.\& Lemelin, C. Le financement de la formation post-secondaire. Revue canadienne de l'enseignement supérieur, 1977, 7 (2), 11-21.

Florian, M. \& Guérin, G. Modèle de prévision de population étudiante GRESIGU. Rapport technique no. 6, Montréal: Université de Montréal, 1971.

Girard, G. La production des universités québécoises et la population de formation universitaire québécoise. Pour la Commission d'enquête sur la situation de la langue française et sur les droits linguistiques au Québec, Québec: L'Editeur officiel du Québec, 1973, Etude E-5.

Girard, G. Les modeles de flux de transition: leur place en économie, leur structure et application empirique. Thèse de maîtrise, Montréal: Département de sciences économiques, Université de Montréal, 1976. 
Girard, G., Otis, J.C. \& Proulx, N. Eléments pour l'étude du personnel scientifique et technique dans le cadre d'une politique de la recherche scientifique au Québec. Montréal: Société Métreq, 1977, en annexe à Québec, Ministre d'Etat au développement culturel. Pour une politique québécoise de la recherche scientifique. Québec: L'Editeur officiel, 1979.

Girard, G., Otis, J.C. \& Proulx, N. Le stock de ressources humaines hautement qualitiées au Québec et la production des universités québécoises. Montréal: Société Métreq et Office de la langue française, 1978.

Girard, M., Gauthier, H. \& Vinet, A. Les jeunes Québécois et le travail. Québec: Office de planification et de développement du Québec, 1978.

Guérin, G. Bibliographie critique des modèles de prévision des effectifs étudiants au niveau universitaire. STOA, 1974, 4 (1), 55-68.

Guérin, G. Elaboration d'un modèle de prévision des effectifs étudiants au niveau universitaire. Thèse de doctorat, Montréal: Départment d'informatique, Université de Montréal, 1973.

Guérin, G. Eléments d'un modèle global de planification des effectifs étudiants au niveau universitaire. Revue canadienne de l'enseignement supérieur, 1975, 5 (2), 9-22.

Guérin, G. Formation-emploi des diplômés en relations industrielles, étude de cas, l'Université de Montréal. Relations Industrielles, 1979, 34 (4), 740-767.

Houle, R. \& Ouellet, L. L'influence des facteurs socio-économiques sur la demande privée pour l'éducation universitaire. Montréal: Département des sciences économi ques, Université de Montréal, 1981.

I.I.E.Q. La population des diplômés et les diplômés en sciences appliquées au Québec. Etude particulière no. 1, pour la Commission d'enquête sur la situation de la langue française et sur les droits linguistiques \& pour la DGES, Montréal: IIEQ, 1971.

Lacroix, R. \& Lemeli n, C. Education supérieure et revenu. in Conseil Economique du Canada. Observations sur les revenus au Canada. Ottawa: Conseil Economique du Canada, 1980.

Lacroix, R., Lemelin, C. \& Robillard, P. Champ de spécialisation et revenu. Actualité Economique, 1978, 54 (1), 5-20.

Lacroix, R. \& Proulx, M. Une évaluation partielle des pertes ou des gains des provinces résultant de la mobilité des étudiants et diplômés universitaires. Actualité Economique, 1973, 49 (3), 379-402.

Lacroix, R. \& Vaillancourt, F. Attributs linguistiques et disparités de revenus au sein de la main-d'oeuvre hautement qualifiée au Québec. Québec: Conseil de la langue française, 1980.

Lapointe, S. La planification sectorielle: l'opération sciences fondamentales. Revue canadienne de l'enseignement supérieur, 1976, 6 (3), 43-49.

Légaré, J. Aspects démographiques de la planification de l'enseignement. Actualité Economique, 1971, 47 (1), 74-83.

Légaré, J. Démométrie et planification des ressources humaines. Québec: Les Presses de l'Université Laval, 1972.

Lemelin, C. La pratique de l'économie de l'éducation dans le Québec francophone de la dernière décennie (1970-1980): une présentation bibliographique. Montréal: Département de science économique, Université du Québec à Montréal, 1981.

Lemelin, C. La répartition des coûts de l'enseignement universitaire. Coll. dossiers, Québec: Conseil des Universités, Gouvernement du Québec, 1980.

Lemelin, C. Le chômage des nouveaux diplômés et la planification de l'enseignement. in 
Chômage des nouveaux diplômés: un problème social. Montréal: Caron-Lahaie, Charlebois et Associés, 1979.

Lemelin, C. L'éducation et le développement des ressources humaines au Québec. in Montmarquette, C. (éd.). Economie du Québec et choix politiques. Montréal: Presses de l'Université du Québec, 1979.

Lemelin, C. \& Otis, J.C. La théorie économique des choix de carrières: une interprétation et une vérification empirique. Actualité Economique, 1978, 54 (3), 337-354.

Lizée, R.R. \& Lizée, N. La démocratisation de l'éducation au Québec, une étude de cas en Amérique du Nord. Etude préparée pour l'UNESCO, Montréal: Université du Québec à Montréal, 1978.

Marois, J. Impact de la syndicalisation des professeurs d'université sur la structure de rémunération. Thèse de maîtrise, Montréal: Département de sciences économiques, Université de Montréal, 1981.

Migué, J.L. Le nationalisme, l'unité nationale et la théorie économique de l'information. Revue canadienne d'économique, 1970, 3 (2), 183-198.

Otis, J.C. Théorie économique du choix de carrière: Une vérification empirique de l'influence des facteurs économiques sur le choix de carrière. Mémoire de maîtrise, Montréal: Département de sciences économiques, Université de Montréal, 1976.

Polèse, M. \& Léger, J. L'impact des universités sur le développement régional. Coll. dossiers, Québec: Conseil des Universités, Gouvernement du Québec, 1980.

Proulx, P.P. Réflexions sur les facteurs déterminants de l'évolution du financement de l'enseignement supérieur au Canada dans les années 1970 et 1980. Stoa, 1973, 3 (2), 151-156.

Québec, Conseil des Universités. Objectifs généraux de l'enseignement supérieur et grandes orientations des établissements. Sainte-Foy: Conseil des Universités, 1972, 1973 et 1976 , quatre cahiers.

Québec, Ministère de l'Education, Direction Générale de 1'Enseignement Supérieur. Analyse de l'évolution de l'enseignement supérieur à la lumière d'indicateurs socio-économiques. Québec: Ministère de l'Education du Québec, 1980.

Québec, Ministère de l'Education, Opération Sciences Appliquées. Rapport final. Québec, 1973.

Québec, Ministère de l'Education, Opération Sciences Fondamentales. Projet de rapport. Québec, 1975.

Québec, Ministère de l'Education, Opération Sciences de la Santé. Rapport final. Québec, 1976, 2 volumes.

Québec, Ministère de l'Education, Secteur de la Planification. La recherche ASOPE à michemin, promesses et réalisations. Québec, Ministère de l'Education du Québec, 1979.

Raynauld, A. Les incidences économiques de l'instauration d'un régime d'éducation permanente. in G. Daoust (éd) L'éducation permanente et l'université québécoise. Montréal: Presses de l'Université de Montréal, 1975.

Raynauld, A. \& Marion, G. Une analyse économique de la disparité interethnique du revenu. Revue Economique, 1972, 23 (1), 1-19.

Roy, P.M. Le syndicalisme professoral dans l'université. in Hurtubise, R. (éd.) L'Université québécoise du proche avenir. Montréal: Hurtubise HMH, 1973.

Saint-Laurent, P. Les diplômés et l'éducation supérieure: quelques constatations. in Chômage des nouveaux diplômés: un problème social. Montréal: Caron-Lahaie, Charlebois et Associés, 1979. 
15 L'économie de l'enseignement supérieur au Québec au cours de la dernière décennie

Tremblay, Y. Les disparités de salaire au sein de la main-d'oeuvre hautement qualifiée du Canada en 1973. Thèse de maîtrise, Montréal: Département de sciences économiques, Université de Montréal, 1976.

Vallerand, N. Modèle d'évaluation des coûts des programmes: vue générale. Revue canadienne de l'enseignement supérieur, 1977, 7 (2), 39-51.

Vergès-Escuin, R. Enseignement de l'architecture et marché du travail. Revue canadienne de l'enseignement supérieur, 1977, 7 (3), 23-38.

Un collectif d'universitaires. Le syndicalisme universitaire et l'Etat. Montréal: Hurtubise HMH, 1977. 